\title{
Responsiveness comparison between a lift-type and drag-type rotor in waves
}

\author{
Yingchen Yang ${ }^{1}$, Fredrick Jenet ${ }^{1}$, Deyanira Jimenez ${ }^{2}$, Brandon Benavides ${ }^{2}$, Jesus Cerda ${ }^{2}$, and Angel Lopez ${ }^{2}$ \\ ${ }^{1}$ University of Texas Rio Grande Valley, Brownsville·Edinburg.Harlingen, TX, USA \\ ${ }^{2}$ Instituto Tecnológico de Matamoros, Matamoros, Mexico
}

\begin{abstract}
Our recent progress on development of a vertical-axis unidirectional rotary wave energy converter (WEC) is discussed in this work. The WEC features a vertical-axis rotor that preforms unidirectional rotation in waves. The vertical axis arrangement makes the WEC respond well to waves from any direction with no realignment needs. And, the unidirectional behavior of the rotor promises no wavefrequency discrimination, which is in comparison to reciprocating WECs that employ the resonant principle and are very frequency-specific. In our earlier proof-of-concept studies, we have successfully demonstrated two types of rotor designs: a lift type employing hydrofoil blades and a drag type using cup blades. In the present work, the two rotor types were further explored experimentally by employing more rotor configurations and blade shapes. The focus was on revealing the rotor responsiveness in simulated waves under a freewheeling condition. The experimental results were compared between a lift-type and drag-type rotor. The comparison provided in-depth understanding on common features of the two rotor types and major differences between them. The yielded research findings will directly guide the development of a prototype vertical-axis unidirectional WEC.
\end{abstract}

\section{Introduction}

In a huge variety of wave energy converter (WEC) designs, a wave-driven reciprocating motion of a WEC (e.g., heaving up and down, pitching/rocking back and forth, bending/curling on and on, etc.) has been a main theme [1]. Such WEC designs take the advantage of the resonant principle to gain a high efficiency in a favorable range of the wave frequency. For power take-off, many reciprocating WEC designs employ either hydraulic circuits (e.g., Pelamis Wave Power) or other mechanical means $[2,3]$ to convert the WEC's primary reciprocating motion into a secondary unidirectional rotation to be transmitted to a traditional electric generator. Some other WEC designs use a direct electric generator to avoid this type of conversion [4].

Differing from the reciprocating WECs, unidirectional WECs feature unidirectional rotation directly driven by waves [5,6]. This feature makes unidirectional WECs fit well in a broad range of the wave frequency since they do not rely on the resonant principle. The unidirectional rotation could be either about a horizontal axis [5] or about a vertical axis [6]. A horizontal-axis unidirectional WEC demands to have its axis approximately aligned along the wave crest direction. Therefore, if the wave direction changes, WEC realignment is needed. In contrast, a vertical-axis unidirectional WEC is insensitive to the wave direction and, thus, has no needs for realignment.
The undoubted tolerance to the wave frequency and wave propagation direction makes the vertical-axis WEC class very attractive. A successful design of this type would demonstrate a great potential towards a simple and robust WEC for low-cost electricity. Due to the complex flow condition resulted by waves, however, it is a great challenge to realize and optimize such a WEC design. In waves, water particles perform orbital motion with drift $[7,8]$. Therefore, wave-driven water flow is unsteady and omnidirectional. In pursuit of a verticalaxis unidirectional rotor, the omnidirectional water flow is expected to drive the rotor for unidirectional rotation. From the hydrodynamics perspective, it would be ideal if a local flow in any spatial direction could always produce a strong torque along a predefined direction of the vertical axis to drive the rotor for unidirectional rotation. This way, the rotor would be capable of unconditionally absorbing energy from waves in a relatively consistent rather than an intermittent way.

Recently the authors have conducted proof-ofconcept studies on a few vertical-axis WEC designs $[9,10]$. The present work focuses on characterization and comparison of two typical rotor types-a drag type and a lift type - in a freewheeling mode by experimental means. For each rotor type, various blade shapes are explored and the formed rotors are examined in various periodic and directional flows. The main objective is to quantify the unidirectionality of the rotors' angular velocity in response to the above flows in terms of the averaged values, time traces, and amplitude spectra. 


\section{Experimental system}

The experiments were carried out in a wave flume of inner dimensions $15 \mathrm{~m}(\mathrm{~L}) \times 1 \mathrm{~m}(\mathrm{~W}) \times 1.3 \mathrm{~m}(\mathrm{H})$. To characterize a rotor's unidirectional responsiveness to omnidirectional flows, a servo-motor-driven translation carriage developed in hous $\mathrm{e}^{11}$ was employed to translate the rotor in quiescent water in the flume. The carriage can translate the rotor in three modes while maintaining the rotor axis/shaft vertical all the time and allowing the rotor to rotate freely about the shaft. The three modes include horizontal oscillation, vertical oscillation, and circular motion in a vertical plane. The circular motion of the rotor in quiescent water approximately simulates a stationary rotor (with one degree of freedom about the shaft) in deep waves where the water particles perform a circular motion (with Stokes drift neglected) [7]. The horizontal and vertical oscillations represent two components of the circular motion. These two component flow conditions are intended for examining the rotor's responsiveness to flows along the two directions. Using the translation carriage to move the rotor in quiescent water makes it much easier to create relative flows along different directions and vary wave parameters than exposing the rotor in waves. For this reason, no real waves were employed in this study. The frequency and peak-to-peak amplitude associated with the three motion modes are herein defined as the simulated wave frequency $f_{W}$ and simulated wave height $H$. The experimental system with a drag-type rotor on is depicted in figure 1.

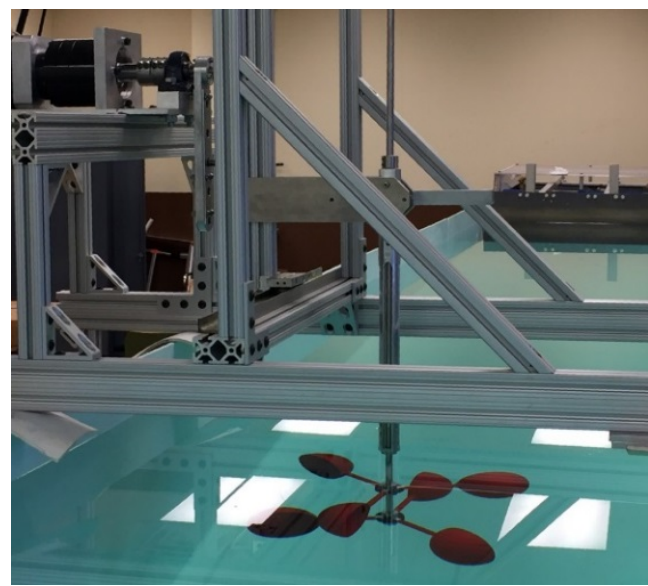

Fig. 1. A photo of the experimental system.

Two types of rotors were examined and compared. They were a drag type and a lift type, (figure 2). A dragtype rotor (figure 2a) employs cup-shaped blades, whereas a lift-type rotor uses wing-shaped blades (figure $2 \mathrm{~b}$ ); all the blades were 3D printed, polished, and painted. To make the two rotor types comparable, some common constraints were applied to all the rotor designs. First, each type consists of two sets of triple blades. Within a set, the three blades show an even circumferential distribution. Between the two sets, there is an angular offset about the shaft and a distance along the shaft to be $60^{\circ}$ and $152.4 \mathrm{~mm}$, respectively. Secondly, both types have the same rotor diameter $D=500 \mathrm{~mm}$. The rotor diameter $D$ is the diameter of the maximum circular swept area of the blades when rotating about the shaft.

For each rotor type, blades with various
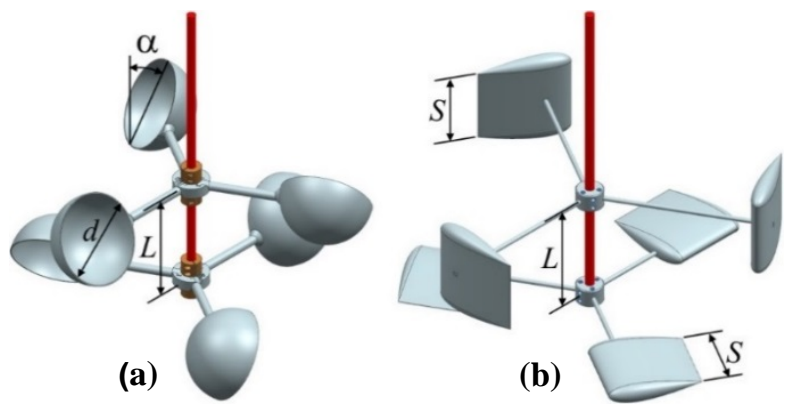

Fig. 2. A drag-type rotor (a) and a lift-type rotor (b).

hydrodynamic shapes were considered. Specifically, in the drag-type rotor design, two cup shapes were employed as blades (figure 3). One was a hemispherical cup and the other a NACA0035-nose cup. Both cups had the same open-end outer diameter of $d=127 \mathrm{~mm}$ and the same wall thickness of $1.5 \mathrm{~mm}$. The NACA0035-nose cup was formed by revolving a NACA0035 profile of a chord length $363 \mathrm{~mm}$ about the chord line and trimming at a chord-wise distance of $108.9 \mathrm{~mm}$ from the nose. Four drag-type rotors were configured using these two types of cup blades: Semi- $0^{\circ}$ (six hemispherical cups with no deflection angle, $\alpha=0^{\circ}$ ), Semi- $30^{\circ}$ (six hemispherical cups with a deflection angle, $\alpha=30^{\circ}$, figure 2a), NACA- $0^{\circ}$ (six NACA0035-nose cups with no deflection angle, $\alpha=0^{\circ}$ ), and NACA-30 ${ }^{\circ}$ (six NACA0035-nose cups with a deflection angle, $\alpha=30^{\circ}$, similar to figure 2a).
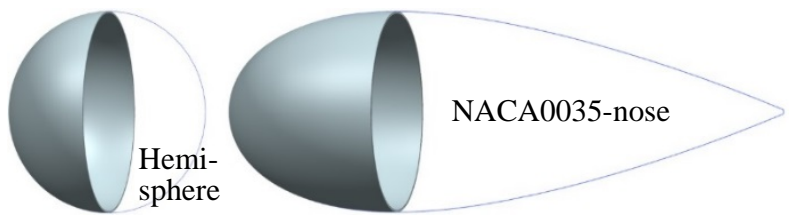

Fig. 3. Two cup shapes for drag-type rotors.

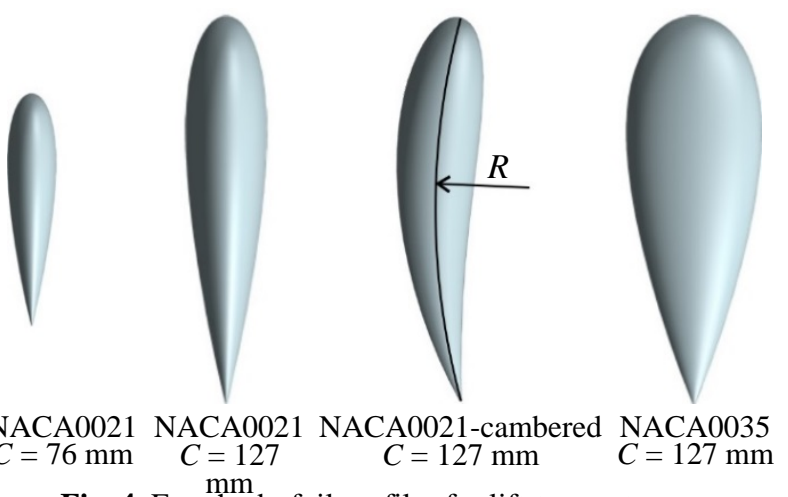

Fig. 4. Four hydrofoil profiles for lift-type rotors.

In the lift-type rotor design, four hydrofoil profiles as specified in figure 4 were adopted. The NACA0021cambered profile had a circular camber line of radius $R=$ $236.7 \mathrm{~mm}$. By extruding each of the four 2-D profiles along the third dimension and rounding both ends to 
form a wing-like blade, all the four blade shapes had the same span of $S=101.6 \mathrm{~mm}$ (excluding the rounded ends, figure $2 \mathrm{~b}$ ). Four lift-type rotors were configured using these four blade shapes: NACA0021-small (six NACA0021 blades with a chord length of $C=76 \mathrm{~mm}$ ), NACA0021 (six NACA0021 blades with $C=127 \mathrm{~mm}$, figure 2b), NACA0021-cambered (three horizontal NACA0021 blades and three vertical NACA0021cambered blades, all with $C=127 \mathrm{~mm}$ ), and NACA0035 (six NACA0035 blades with $C=127 \mathrm{~mm}$ ).

By mounting a rotor to the aforementioned translation carriage through a shaft-bearing-housing mechanism, the rotor can freely rotate about its shaft with low mechanical friction. If there is no power takeoff from the rotor, such a rotation condition is termed as freewheeling. All the four drag-type rotors and four lifttype rotors were tested in a freewheeling mode. And, they were all tested in the same range of the simulated wave frequency $0.33 \leq f_{W} \leq 0.83$ at a fixed wave height $H / D=0.635$. For each experimental run, a 75-second video was taken to record a rotor's real-time angular displacement. A dial that rotated together with the rotor was employed for this purpose. The video was then processed to derive the time trace of the rotor's angular velocity. Details on data acquisition and post processing to obtain time traces were discussed in our earlier work ${ }^{11}$. To determine an averaged angular velocity and standard deviation, three repetitive runs were conducted for each experimental condition considered. The standard deviation turned out to be very low due to the relatively long recording time of individual runs. Therefore, no error bars were provided when presenting the averaged angular velocity.

\section{Results and discussion}

Aiming at characterizing vertical-axis rotors' unidirectional behavior in omnidirectional flows and comparing the two rotor types, experimental results are sorted by the rotor type first and a comparative discussion is provided towards the end.

\subsection{Drag-type rotors}

For the four drag-type rotors (Hemi- $0^{\circ}$, Hemi-30 ${ }^{\circ}$, NACA $-0^{\circ}$, and NACA $-30^{\circ}$ ) in three types of the carrying motion (circular motion, horizontal oscillation, and vertical oscillation), the averaged angular velocity $\bar{\omega}$ versus the simulated wave frequency $f_{W}$ are presented in figure 5. In circular carrying motion, figure 5a shows an approximately linear trend of $\bar{\omega} \sim f_{W}$ for each rotor, and no significant difference among the four rotors is observed. A couple of noticeable differences are still evident though: (i) at a fixed deflection angle $\left(0^{\circ}\right.$ or $\left.30^{\circ}\right)$, a rotor with hemispherical blades has slightly higher $\bar{\omega}$ than a rotor with NACA-nose blades and, (ii) with a selected blade shape (hemisphere or NACA nose), a rotor with a $30^{\circ}$ deflection angle resulted in a somewhat higher $\bar{\omega}$ than a rotor with a $0^{\circ}$ deflection angle.
It is known from cup anemometer study [12] that a cup rotor with a $0^{\circ}$ deflection angle responds well with flows normal to the rotor shaft. The employment of a $30^{\circ}$ angle is intended to enhance the rotor responsiveness to flows along the shaft direction [9]. Figure 5a indirectly supports this intention. More insight is gained from figure $5 c$ for rotors in vertical oscillatory flows. Specifically, between $\mathrm{Hemi}-0^{\circ}$ and $\mathrm{Hemi}-30^{\circ}$ the deflection only leads to a minor increase in $\bar{\omega}$ in relatively high values of $f_{W}$. Between NACA- $0^{\circ}$ and NACA- $30^{\circ}$, however, a large increase has been observed, and it gets stronger with the increasing $f_{W}$. It is noteworthy though, that such enhancement to vertical flows is achieved by sacrificing the rotor responsiveness to horizontal flows, as evident in figure $5 \mathrm{~b}$. It is particular severe on the rotor NACA-30 ${ }^{\circ}$; it demonstrates the strongest rotation among the four rotors in vertical oscillatory flows (figure 5c) but the weakest in horizontal oscillatory flows (figure 5b). Further
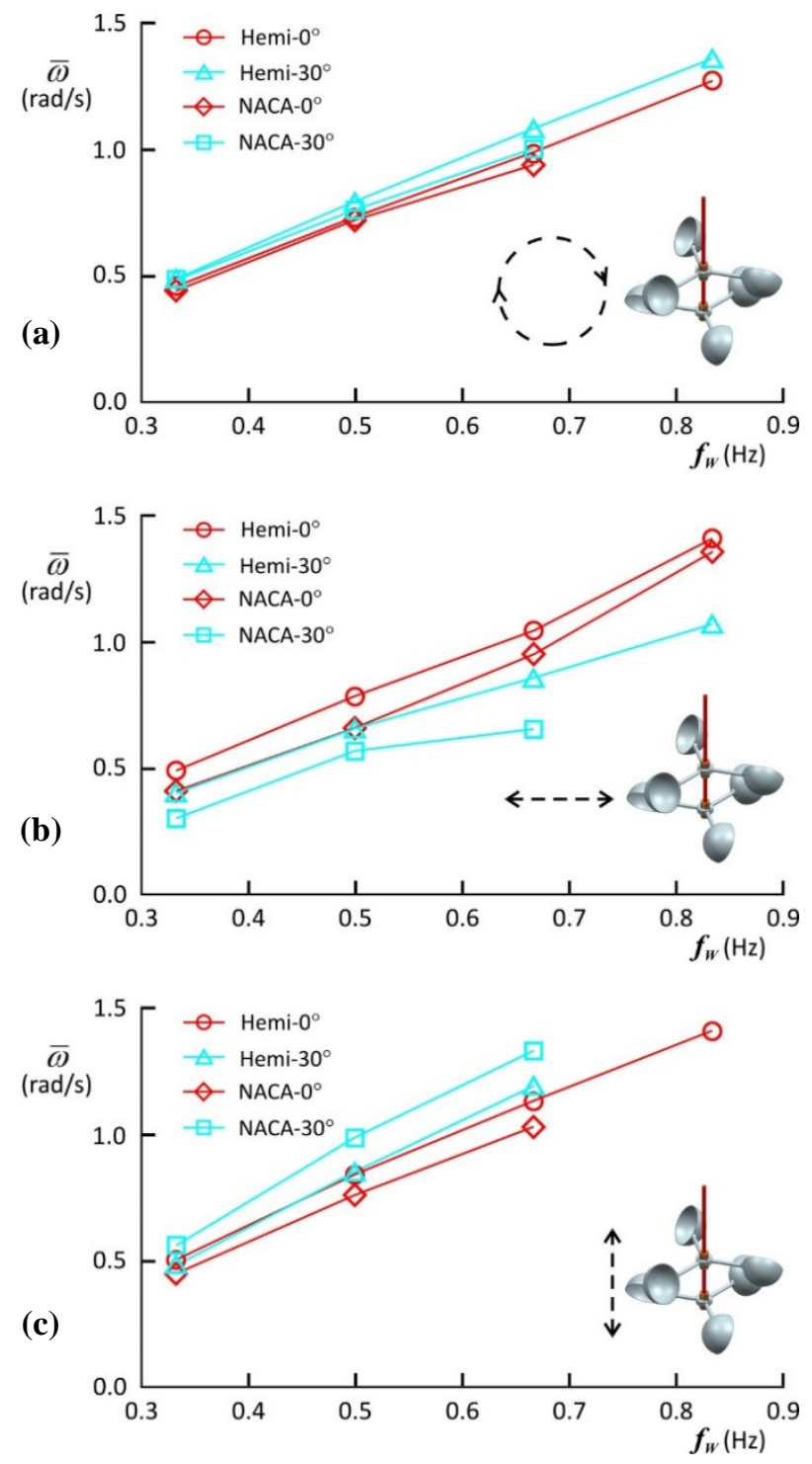

Fig. 5. Averaged angular velocity $\bar{\omega}$ versus the simulated wave frequency $f_{W}$ for four drag-type rotors in three carrying motion types. (a) Circular motion. (b) Horizontal oscillation. (c) Vertical oscillation. 
comparison between figures $5 \mathrm{~b}$ and $5 \mathrm{c}$ leads to another very interesting observation: both the rotors with no deflection (i.e., Hemi- $0^{\circ}$ and NACA- $0^{\circ}$ ) already rotate as well in vertical oscillatory flows as in horizontal oscillatory flows.

Time traces and associated spectra reveal further details on the rotors' unidirectional behavior in response to flows in different directions. Figures 6 through 9 present such results for each of the four drag-type rotors at a selected wave frequency $f_{W}=0.67 \mathrm{~Hz}$. Time $t$ in the time traces is normalized by the wave period $T, T=1 / f_{W}$, and the frequency $f$ in the spectra is normalized by $f_{W}$. Both common features and distinctions are evident as presented by these figures.

With a specified rotor and among the three types of carrying motion, the differences among the time traces are easy to tell in terms of the waveforms and levels of fluctuation (e.g., figures 6a, 6c, and 6e). Such differences are also reflected in the corresponding spectra with different numbers and levels of peaks (e.g., figures 6b, $6 \mathrm{~d}$, and $6 \mathrm{f}$ ). With a specified type of carrying motion and among the four rotors, however, waveform resemblance among the time traces is evident (e.g., figures 6a, 7a, 8a, and 9a) except for the vertical carrying motion. Obviously, in the vertical carrying motion a rotor responsiveness is largely affected by the blade shape and deflection angle (figures 6e, 7e, 8e, and 9e). For all the four rotors and three carrying motion types, an overly dominant peak always occurs at twice the simulated wave frequency in every spectrum. A noticeable peak at the wave frequency does exist in the circular carrying motion (figures 6b, 7b, 8b, and 9b), but is almost negligible in the horizontal and vertical carrying motion. In addition, there are one or two discernible peaks nearby the peak at the wave frequency in the circular
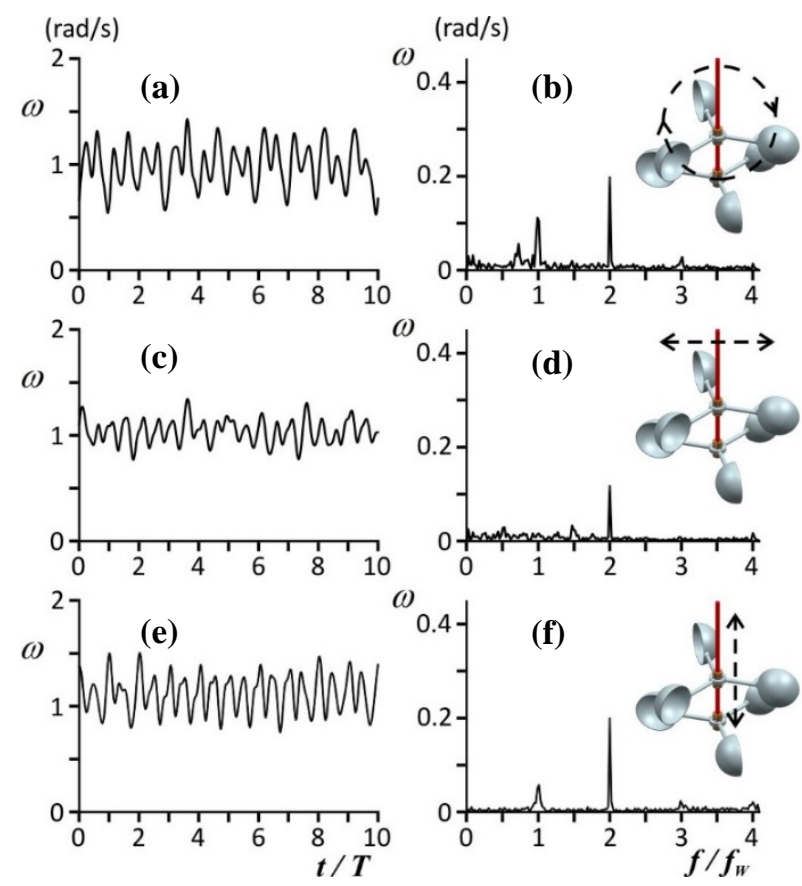

Fig. 6. Time traces (left column) and amplitude spectra (right column) of the Hemi- $0^{\circ}$ rotor's angular velocity in response to three carrying motions as specified by inserts on the right. carrying motion (figures 6b, 7b, 8b, and 9b), and the physics is unclear yet.

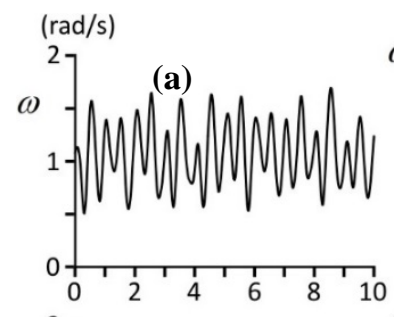

$(\mathrm{rad} / \mathrm{s})$
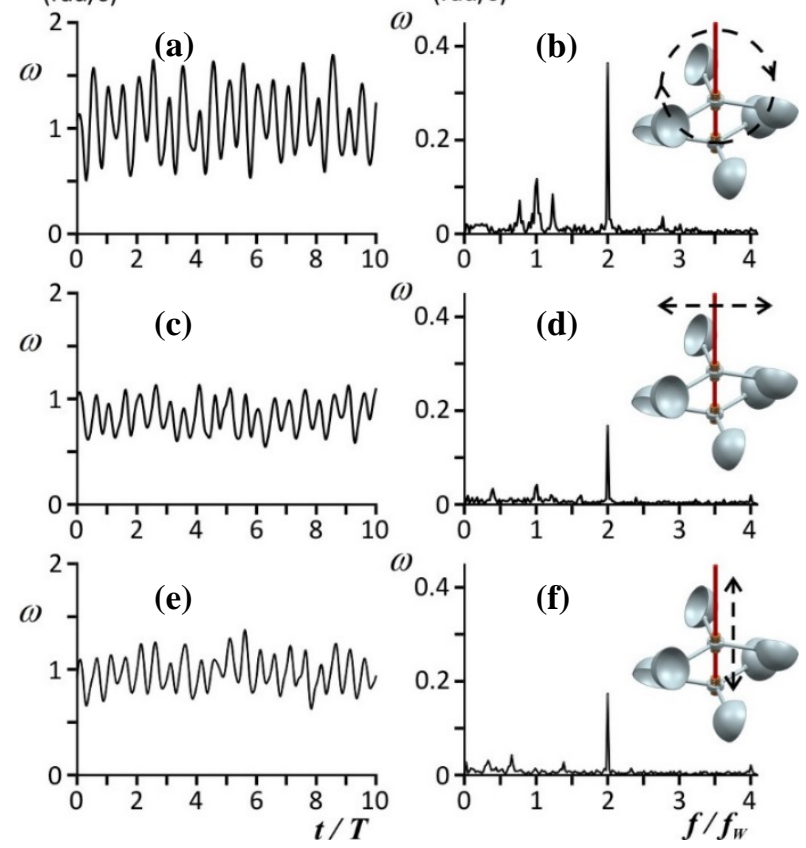

Fig. 7. Time traces (left column) and amplitude spectra (right column) of the Hemi- $30^{\circ}$ rotor's angular velocity in response to three carrying motions as specified by inserts on the right.
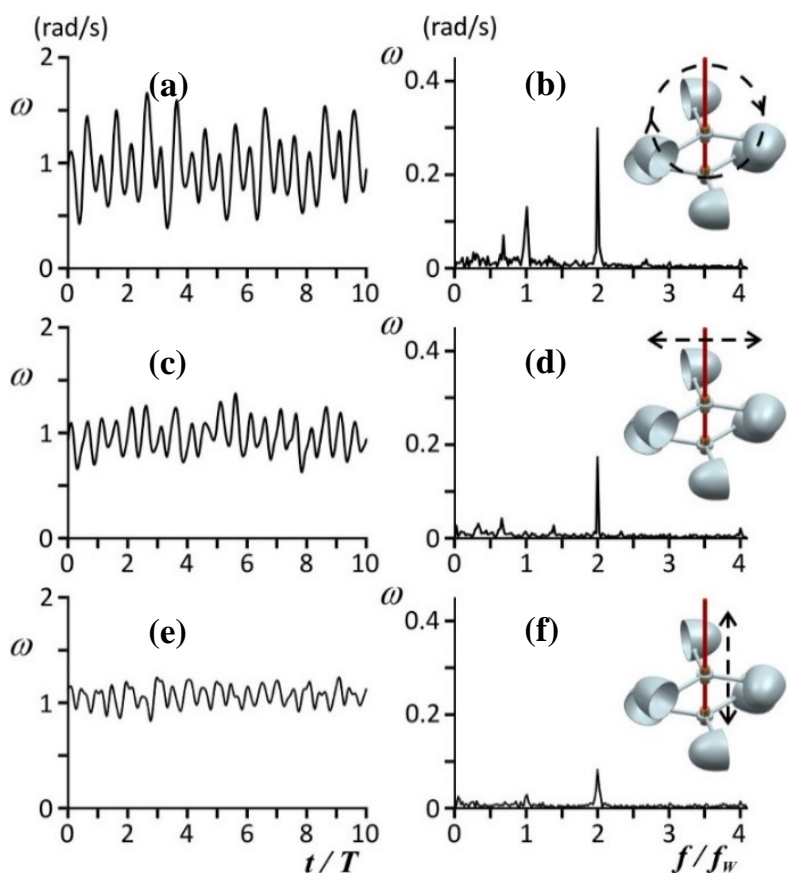

Fig. 8. Time traces (left column) and amplitude spectra (right column) of the NACA- $0^{\circ}$ rotor's angular velocity in response to three carrying motions as specified by inserts on the right.

\subsection{Lift-type rotors}

The averaged angular velocity $\bar{\omega}$ versus the simulated wave frequency $f_{W}$ for the four lift-type rotors (NACA0021, NACA0021-cambered, NACA0021-small, 

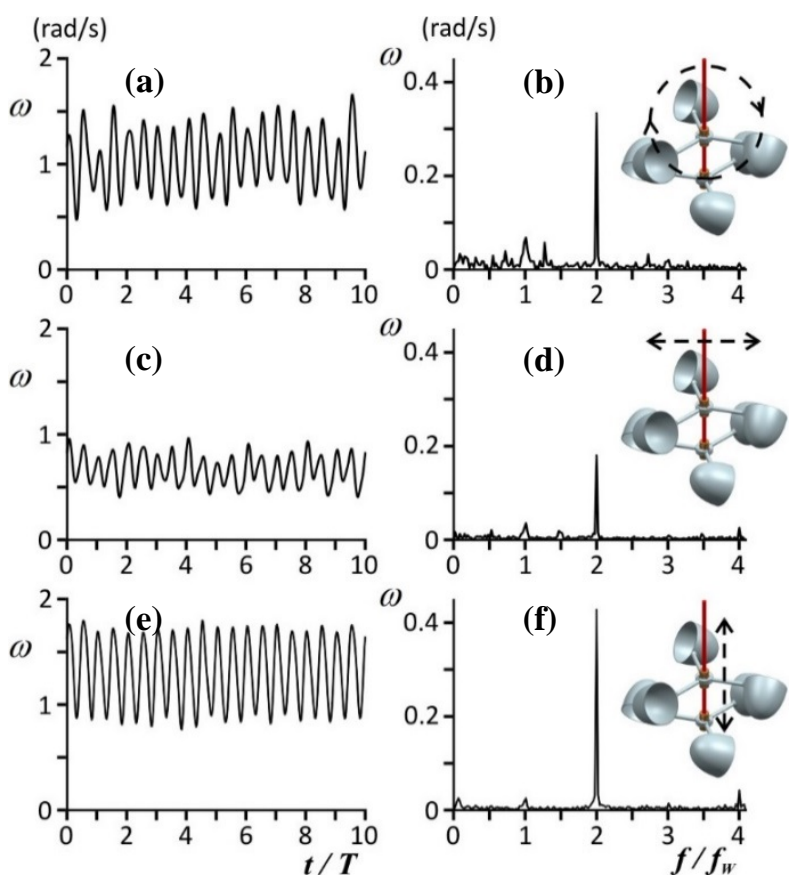

Fig. 9. Time traces (left column) and amplitude spectra (right column) of the NACA- $30^{\circ}$ rotor's angular velocity in response to three carrying motions as specified by inserts on the right.

and NACA0035) are presented in figure 10. Specifically, figures 10a, 10b, and 10c compare the four rotors in circular, horizontal, and vertical carrying motions, respectively. A general pattern in figure 10 is that $\bar{\omega}$ increases with $f_{W}$ for each case considered. For different rotors in different carrying motions, however, individual trends are quite different. Another common behavior is that, the four rotors all demonstrate a lower $\bar{\omega}$ in the horizontal carrying motion than in the circular or vertical ones. Such a weak responsiveness to horizontal flows can be addressed by properly increasing the span of the three vertical blades in a rotor design.

Among the four rotor designs, comparing NACA0021 with NACA0021-small provides understanding on how the blade chord length $(127 \mathrm{~mm}$ versus $76 \mathrm{~mm}$ ) changes a rotor's rotation. Similarly, comparing NACA0021 with NACA0035 helps quantify the effect of the blade thickness $(26.7 \mathrm{~mm}$ versus 44.5 $\mathrm{mm}$ ), and comparing NACA0021 with NACA0021cambered allows determination of the blade cambering effect (straight versus cambered).

As shown in figure10, an increase in the chord length (from NACA0021-small to NACA 0021) leads to a quite noticeable increase in $\bar{\omega}$ in all the three carrying motions. In contrast, an increase in the blade thickness (from NACA0021 to NACA0035) results in a slight decrease in $\bar{\omega}$ in circular motion, a negligible change in horizontal oscillation, and a relatively large increase in vertical oscillation at lower values of $f_{w}$. The most remarkable difference is made by the blade cambering (from NACA0021 to NACA0021-cambered); a significant increase in $\bar{\omega}$ has been achieved in all the three carrying motions, and the increase enhances with increasing $f_{W}$.
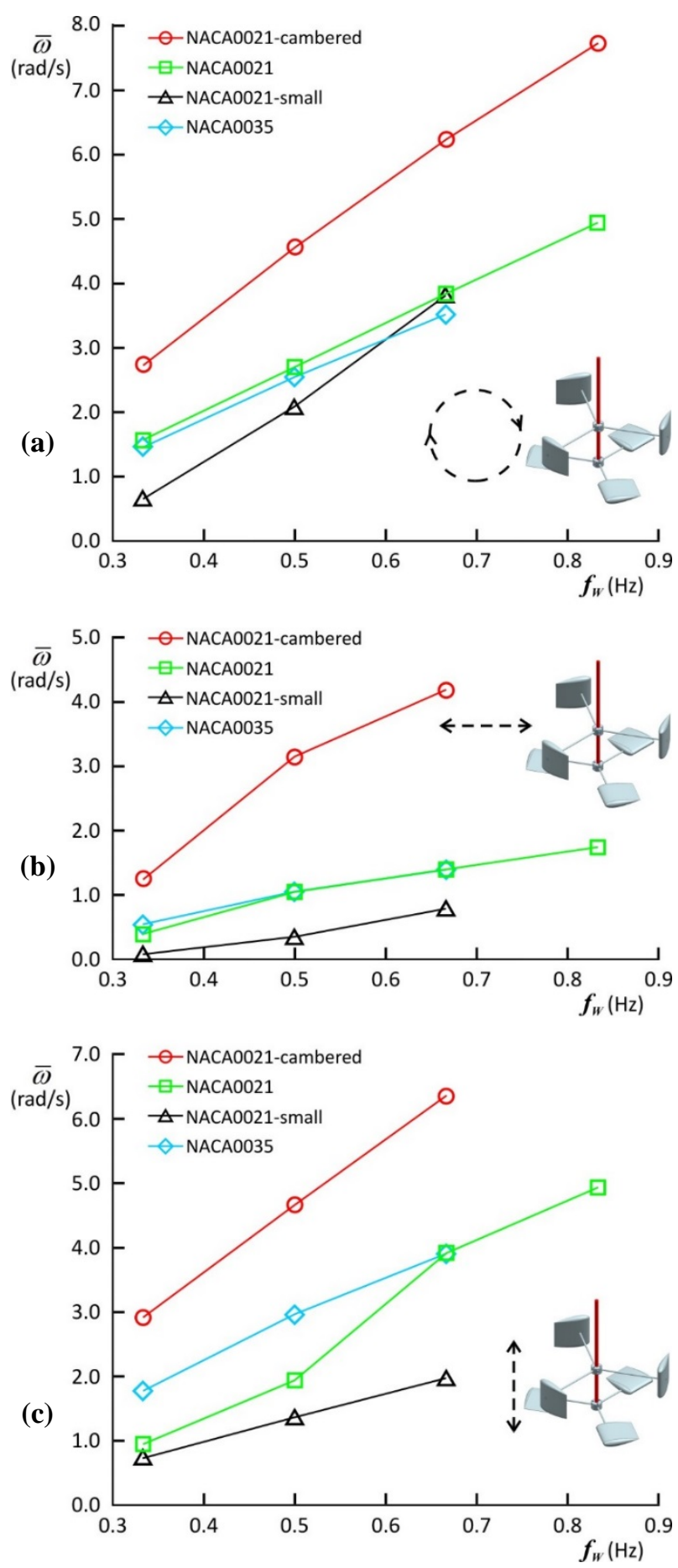

Fig. 10. Averaged angular velocity $\bar{\omega}$ versus the simulated wave frequency $f_{W}$ for four lift-type rotors in three carrying motion types. (a) Circular motion. (b) Horizontal oscillation. (c) Vertical oscillation.

Overall, the NACA0021-cambered rotor demonstrates the best performance among the four rotors.

Time traces and spectra for the four lift-type rotors in three carrying motions and at a selected wave frequency $f_{W}=0.67 \mathrm{~Hz}$ are shown in figures 11 through 14 . Note that all the time traces are plotted in the same scale but in various ranges of $\omega$.

Similar to the drag type-rotors, the lift-type rotors yield time traces in different waveforms and fluctuation levels in response to different carrying motions. For 

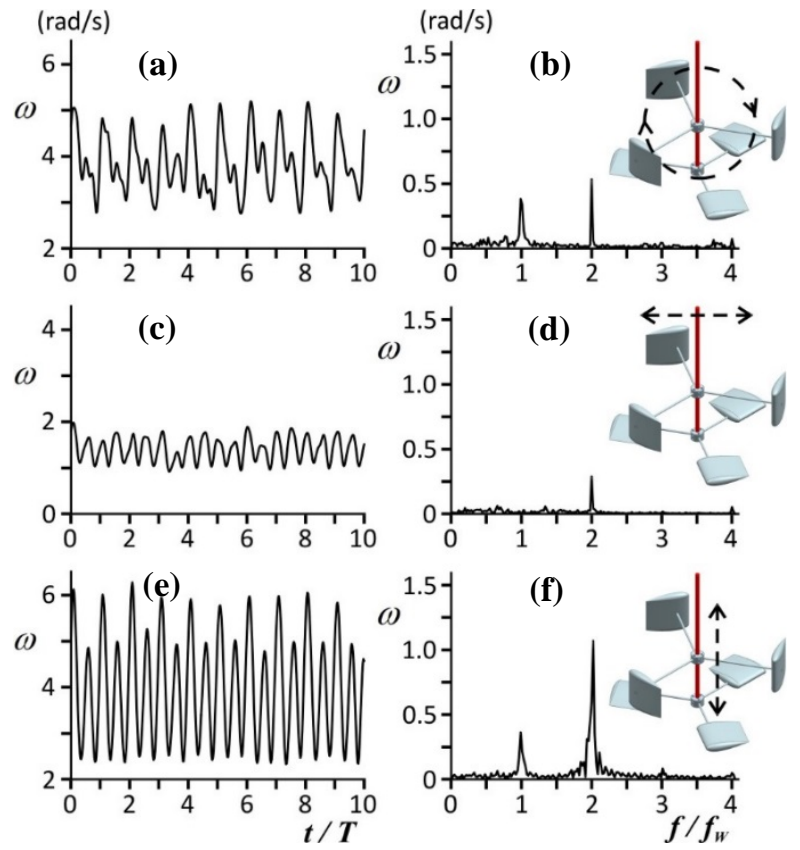

Fig. 11. Time traces (left column) and amplitude spectra (right column) of the NACA0021 rotor's angular velocity in response to three carrying motions as specified by three inserts on the right.
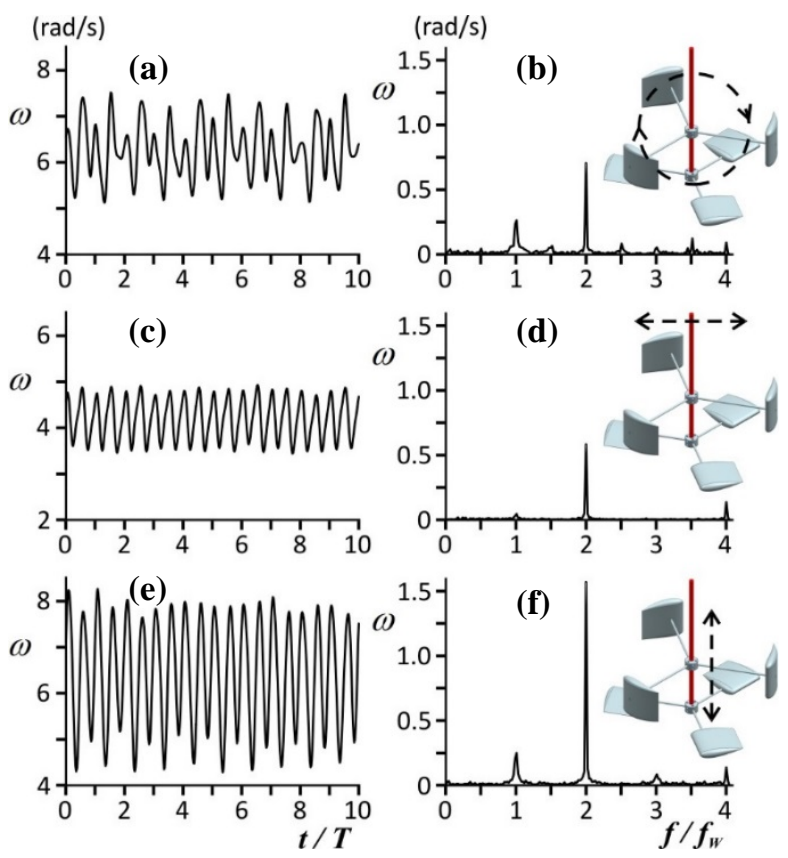

Fig. 12. Time traces (left column) and amplitude spectra (right column) of the NACA0021-cambered rotor's angular velocity in three carrying motions as specified by three inserts on the right.

example, in the vertical carrying motion, both the rotors NACA0021 and NACA0021-cambered gain a strong rotation in terms of the mean value and fluctuation of $\omega$ (figures 11e and 12e). In the horizontal carrying motion, however, the mean value and fluctuation drops largely (figures 11c and 12c). Comparison between figures 11 and 12 shows that, both the horizontal and vertical carrying motions result in time traces close to a rectified waveform with an offset for the NACA0021 rotor

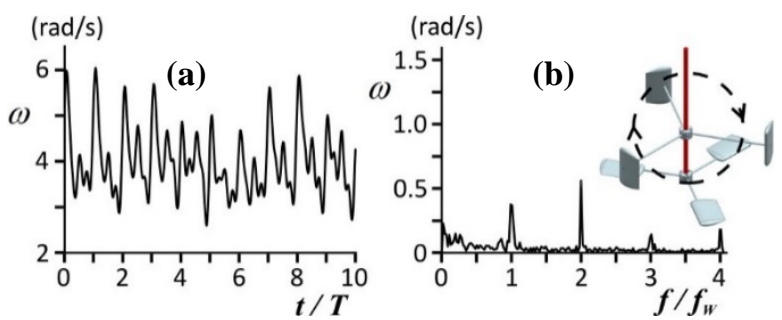

Fig. 13. Time traces (left) and amplitude spectra (right) of the NACA0021-small rotor's angular velocity in response to the circular carrying motion as specified by the insert on the right.
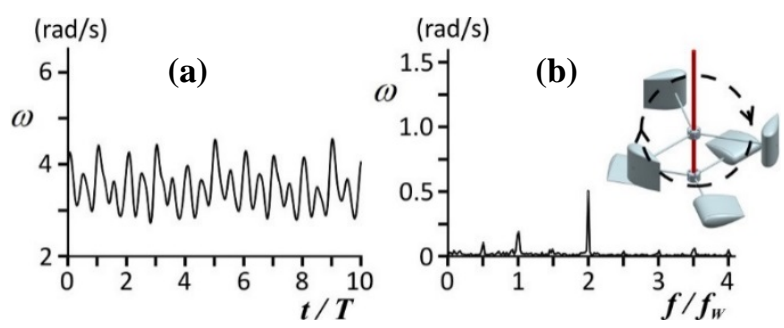

Fig. 14. Time traces (left) and amplitude spectra (right) of the NACA0035 rotor's angular velocity in response to the circular carrying motion as specified by the insert on the right.

(figures 11c and 11e), but it is not the case for the NACA0021-cambered (figures $12 \mathrm{c}$ and 12e). The waveforms in the circular motion for the lift-type rotors are quite similar (figures 11a, 12a, and 14a). They also resemble that for the drag-type rotors (figures $6 \mathrm{a}, 7 \mathrm{a}, 8 \mathrm{a}$, and 9a) to a large extent. An exception is the NACA0021-small rotor in the circular carrying motion; the time trace (figure 13a) is much noisier than others. The corresponding spectrum (figure 13b) shows a dominant peak at $f / f_{W}=2$ and a less dominant peak at $f / f_{W}=1$. The noise is due to the discernable higher harmonics at $f / f_{W}=3$ and 4 , as well as some very low frequency interference.

The spectra in figures 11 through 14 all present a dominant peak at $f / f_{W}=2$, which is, once again, similar to that for drag-type rotors. In contrast, the secondary peak at $f / f_{W}=1$ is comparatively small in circular motion (figures 11a, 12a, 13a, and 14a), insignificant in vertical oscillation, and hardly discernable in horizontal oscillation.

\subsection{Comparison}

Using the averaged angular velocity $\bar{\omega}$ obtained from the circular carrying motion as a criterion, the best performing rotor from each of the two rotor types is selected. One is Hemi- $30^{\circ}$ from the drag type and the other is NACA0021-cambered from the lift-type. The two representative rotors are compared in terms of $\bar{\omega}$, as shown in figure 15. Obviously, under the same flow conditions the lift-type rotor rotates much faster than the drag-type rotor. Specifically, in the circular carrying motion the lift-type rotor rotates about 5.7 times as fast as the drag-type rotor in the whole testing range of $f_{\mathrm{W}}$.

It has long been known that, in uniform flows, a lifttype rotor is generally more efficient than a drag-type 


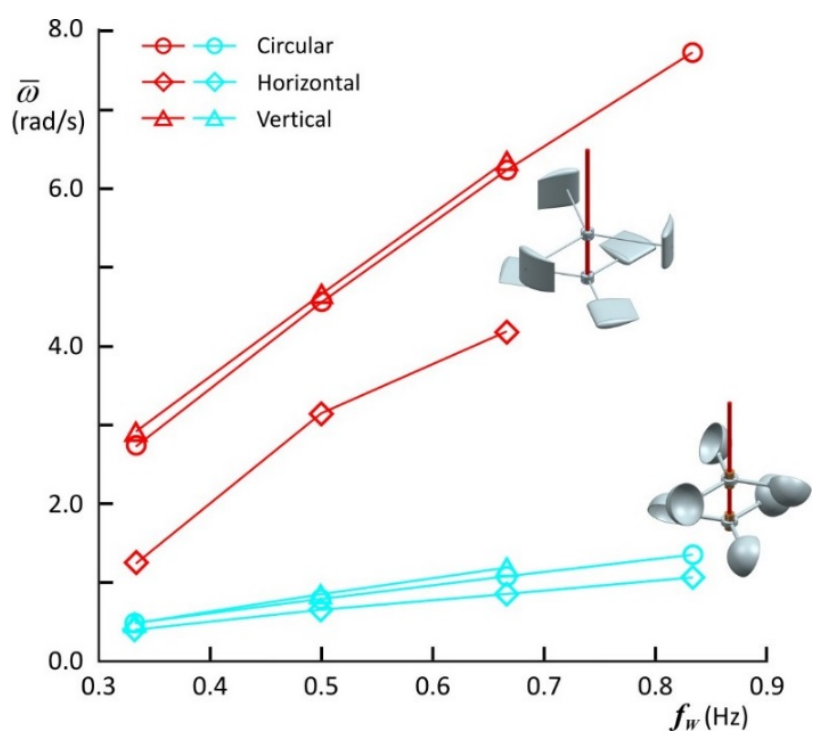

Fig. 15. Comparison of $\bar{\omega}$ between a drag-type rotor (Hemi$30^{\circ}$ ) and a lift-type rotor (NACA0021-cambered) among three motion types.

rotor. The much stronger flow separation in the dragtype rotor results in more loss of the flow's hydrokinetic energy on the viscous dissipation. In wave-rotor interaction, however, this common understanding may need to be re-evaluated. Due to the omnidirectional nature of water flow in waves, an instantaneous angle of attack of a local flow with respect to a lift-type rotor blade could sometimes go very large (e.g., $90^{\circ}$ or beyond), leading to a flow separation as severe as, or even worse than, the drag-type case.

While figure 15 still supports the common understanding, the data were obtained in a freewheeling mode. In this case the lift-type rotor rotates very fast. Hence it might be able to keep the angles of attack of individual blades in waves in a relatively small level. In a power take-off mode, however, the rotor's rotation may need to be reduced to a lower level due to a variety of considerations (e.g., an added resistive load, cavitation avoidance, power output maximization, etc.). Then the slowed rotation may lead to a large increase in the angles of attack and make the rotor loose its advantages. Therefore, a more comprehensive comparison between the two rotor types by employing a power take-off mechanism needs to be considered in future research.

\section{Conclusions}

Two types of vertical-axis unidirectional rotors-a drag type and a lift type-were experimentally explored in simulated waves in a freewheeling mode. In each rotor type, four rotors with different blade shapes and dimensions were employed. The rotors' unidirectional responsiveness was characterized in terms of the time traces, spectra, and averaged values of a rotor's angular velocity. In simulated waves, all the rotors demonstrated highly unidirectional rotation, yet with fluctuation. An overly dominant frequency of the fluctuation occurred at twice the simulated wave frequency for all the rotors.
Two selected rotors, with each representing one rotor type, were compared by means of the averaged angular velocity. The lift-type rotor rotated 5.7 times as fast as the drag-type rotor.

\section{References}

1. M.S. Guney, K. Kaygusuz, Renew. Sust. Energ. Rev. 14, 2996-3004 (2010)

2. J Ai, H. Lee, C. Liang, L. Zuo, Proc. ASME 2014 Int. Design Engineering Technical Conf. V008T11A086 (2014)

3. A. Battezzato, J. Theor. App. Mech., 53, 195-207 (2015)

4. M. Leijon, H. Bernhoff, O. Agren, J. Isberg, J. Sundberg, et al., IEEE Trans. Energy Convers. 20, 219-24 (2005)

5. M.R. Ahmed, M. Faizal, Y.H. Lee, Ocean Eng. 65, 32-8 (2013)

6. E.A. Rossen, P.C. Scheijgrond, R. Mikkelsen, Proc. 4th European wave Energy Conf. 2389448844 (2000)

7. G.L. Pickard, S. Pond Introductory Dynamical Oceanography 2nd ed (Butterworth-Heinemann, 1983)

8. O.M. Phillips, The dynamics of the upper ocean 2nd ed (Cambridge University Press, 1977)

9. Y. Yang, I. Diaz, M. Morales, P. Obregon, Proc. ASME $34^{\text {th }}$ Int. Conf. on Ocean, Offshore and Arctic Engineering doi:10.1115/OMAE2015-41140 (2015)

10. Y. Yang, I. Diaz, S. Soto, Proc. ASME 2016 Int. Mechanical Engineering Congress \& Exposition doi:10.1115/IMECE2016-65952 (2016)

11. Y. Yang, F. Salazar, J. Soto, Proc. ASME 2017 Fluids Engineering Summer Conf. doi:10.1115/FEDSM2017-69458 (2017)

12. S. Pindado, J. Cubas, A. Sanz-Andrés, Sci. World J. 197325 (2013) 